\title{
ONE-DIMENSIONAL LINEAR ANALYSIS OF THE LIQUID INJECTION OR REMOVAL IN A LIQUID BRIDGE
}

\author{
J. Meseguer and A. SAnZ \\ Laboratorio de Aerodinàmica, ETSI Aeronàuticos, Universidad Politécnica, 28040 Madrid, Spain
}

\begin{abstract}
The filling-withdrawal process of a long liquid bridge is analyzed using a one-dimensional linearized model for the dynamics of the liquid column. To carry out this study, a well-known standard operational method (Laplace transform) has been used, and time variation of both liquid velocity field and interface shape are obtained.
\end{abstract}

\section{INTRODUCTION}

In recent years, the knowledge on the behavior of long liquid bridges in a reduced gravity environment has been greatly extended because of the increased possibilities of experimentation with such fluid configurations aboard space platforms[1,2]. Although theoretical studies concerning liquid bridges extend over a wide variety of aspects (a brief scope of the state of the art in this field can be found in Meseguer and Sanz[3]) a considerable effort has been devoted to the analysis of the response of a liquid bridge to mechanical disturbances, particularly to the analysis of liquid bridge stability limits.

The problem of the injection (or removal) of working liquid into a liquid bridge is analyzed in this paper by using a one-dimensional slice linearized model of the liquid bridge dynamics. Such onedimensional liquid bridge model has been used previously in related problems, its ability to predict liquid bridge behavior being demonstrated by the agreement between theoretical and experimental results, the last being obtained either in simulated microgravity $[3,4]$ or aboard Spacelab-D1[5].

The problem to be solved, as sketched in Fig. 1, consists of a liquid column of length $L$ held by surface tension forces between two coaxial, equal diameter, disks of radius $R_{0}$, the liquid injection (or removal) being performed through one of the disks. Injection rate is assumed to be small enough so that interface deformation and velocity field inside the liquid column are also small to allow a linear approach. The linearized formulation has been solved by using well-known Laplace transform techniques for partial differential equations[6,7]. In our study, it is assumed a step function for the liquid injection law so that once the response of the liquid bridge to this stimulus is calculated, the response to any other injection law can be calculated by using convolution properties.

Academy Member (Section 2).

\section{MATHEMATICAL MODEL}

In the following, unless otherwise stated, all physical quantities are made dimensionless using the characteristic length $K_{0}$, the radius of the disks, and the characteristic time $\left(\rho R_{0}^{3} / \sigma\right)^{1 / 2}, \rho$ being the liquid density and $\sigma$ the surface tension.

The one-dimensional slice model can be deduced from Euler equations by assuming the axial velocity $W$ to be dependent upon the axial coordinate $z$ and the time $t$ but not upon the radial coordinate $r$. With this choice, the radial momentum equation becomes decoupled, and the following formulation[8] results Continuity equation

$$
\left(R^{2}\right)_{t}+\left(W R^{2}\right)_{z}=0
$$

Axial momentum equation

$$
W_{t}+W W_{z}=-P_{z}
$$

where the reduced capillary pressure $P$ is given by

$$
P=\left[1+\left(R_{z}\right)^{2}\right]^{-3 / 2}\left[1+\left(R_{z}\right)^{2}-R R_{z z}\right] R^{-1} .
$$

In these expressions $R=R(z, t)$ stands for the equation of the liquid bridge interface. Boundary conditions are

$R( \pm \Lambda, t)=1, W(-\Lambda, t)=0, W(\Lambda, t)=\epsilon S_{0}(t),(4)$

where $A=L /(2 R)$ is the liquid bridge slenderness, $\epsilon$ is a small parameter measuring the liquid injection (or removal) rate (according to Fig. $1 \epsilon>0$ means lic uid removal) and $S_{0}(t)$ stands for the unit step function $\left[\left(S_{k}(x)=0\right.\right.$ when $0<x<k, S_{k}(x)=1$ when $x>k)$ ]. Initial conditions are:

$$
R(z, 0)=1, W(z, 0)=0,
$$

that is, the liquid bridge is initially at rest, its interface being cylindrical.

Then, if $\epsilon$ is small enough, ignoring $\epsilon^{2}$ terms, the variables involved in the problem may be written as

$$
\begin{aligned}
R(z, t)=1+ & \epsilon \tilde{R}(z, t), W(z, t) \\
& =\epsilon \tilde{W}(z, t), P(z, t)=1+\epsilon \tilde{P}(z, t) .
\end{aligned}
$$




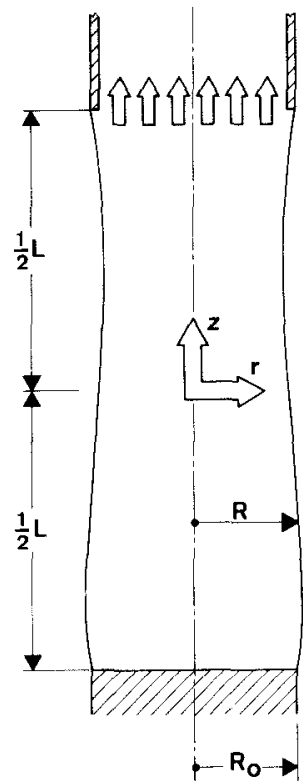

Fig. 1. Geometry and coordinate system for the liquid bridge problem

After substituting these expressions in the above formulation there results

$$
\begin{gathered}
2 \tilde{R}_{t}+\tilde{W}_{z}=0 \\
\tilde{W}_{t}+\tilde{P}_{z}=0
\end{gathered}
$$

In order to simplify expressions a new time variable $\tau=t / \sqrt{2}$ is introduced so that eqn (12) finally results

$$
\tilde{W}_{\tau \tau}+\tilde{W}_{z z}+\tilde{W}_{z z z z}=0 \text {. }
$$

\section{PROBLEM SOLUTION}

Let $w(z, s)$ the Laplace transform of $\tilde{W}(z, \tau)$, then, in view of the initial conditions (14), there results

$$
\begin{gathered}
w_{z z z z}+w_{z z}+s^{2} w=0, \\
w(\Lambda, s)=s^{-1}, w(-\Lambda, s)=0, w_{z}( \pm \Lambda, s)=0
\end{gathered}
$$

The solution of eqn (16) is

$$
\begin{aligned}
w(z, s)=A_{1} \exp (a z)+A_{2} \exp (-a z) & \\
& +A_{3} \exp (b z)+A_{4} \exp (-\mathrm{bz})
\end{aligned}
$$

where $A_{i}$ are complex numbers and

$$
\begin{aligned}
& a=\left\{-\frac{1}{2}\left[1-\left(1-4 s^{2}\right)^{1 / 2}\right]\right\}^{1 / 2}, \\
& b=\left\{-\frac{1}{2}\left[1+\left(1-4 s^{2}\right)^{1 / 2}\right]\right\}^{1 / 2},
\end{aligned}
$$

are the roots $(\theta= \pm a, \theta= \pm b)$ of the characteristic equation

$$
\theta^{4}+\theta^{2}+s^{2}=0 .
$$

The four constants appearing in (18) are calculated by solving the four equations resulting from the fulfillment of the boundary conditions (17)

$$
\left[\begin{array}{cccc}
\exp (a \Lambda) & \exp (-a \Lambda) & \exp (b \Lambda) & \exp (-b \Lambda) \\
\exp (-a \Lambda) & \exp (a \Lambda) & \exp (-b \Lambda) & \exp (b \Lambda) \\
a \exp (a \Lambda) & -a \exp (-a \Lambda) & b \exp (b \Lambda) & -b \exp (-b \Lambda) \\
a \exp (-a \Lambda) & -a \exp (a \Lambda) & b \exp (-b \Lambda) & -b \exp (b \Lambda)
\end{array}\right] \quad\left[\begin{array}{c}
A_{1} \\
A_{2} \\
A_{3} \\
A_{4}
\end{array}\right]=\left[\begin{array}{c}
s^{-1} \\
0 \\
0 \\
0
\end{array}\right]
$$

$$
\tilde{P}=-\tilde{R}-\tilde{R}_{z z},
$$

resulting

$$
\begin{gathered}
\tilde{R}( \pm \Lambda, t)=0, \tilde{W}(-\Lambda, t)=0, \tilde{W}(A, t)=S_{0}(t), \\
\tilde{R}(z, 0)=0, \tilde{W}(z, 0)=0 .
\end{gathered}
$$$$
w(z, s)=\frac{1}{s} \frac{M(z, s)}{D(s)},
$$

Introducing in (8) the perturbation pressure, as given by (9), and eliminating the variable $R$ between (7) and (8), yields

$$
2 \tilde{W}_{u}+\tilde{W}_{z z}+\tilde{W}_{z z z z}=0,
$$

which is the differential equation to be solved, together with boundary and initial conditions

$$
\begin{gathered}
\tilde{W}_{2}( \pm \Lambda, t)=0, \tilde{W}(-\Lambda, t)=0, \\
\tilde{W}(\Lambda, t)=S_{0}(t), \\
\tilde{W}(z, 0)=0, \tilde{W}_{t}(z, 0)=0,
\end{gathered}
$$

where the first boundary condition has been obtained from that expressing the attachment at the disk edges $[\tilde{R}( \pm A, t)=0]$, which states, according to (7) that $W$ must reach its values with zero slope. In a similar way, taking into account eqns (9) and (8), the last initial condition can be deduced. where $D(s)$ stands for the determinant of the system (21)

$D(s)=4[\sinh 2 \mathrm{a} A \sinh 2 b \Lambda$

$$
+2 a b(\cosh 2 a A \cosh 2 b A-1)] \text {, }
$$

and the function $M(z, s)$ is

$$
\begin{aligned}
M(z, s)= & 4\left\{a^{2} \sinh 2 \mathrm{a} A \sinh b(\Lambda+z)\right. \\
& +b^{2} \sinh a(\Lambda+z) \sinh 2 b \Lambda \\
& +\mathrm{ab}[\cosh 2 \mathrm{a} A \cosh b(\Lambda+z) \\
& +\cosh a(\Lambda+z) \cosh 2 b A \\
& -\cosh a(\Lambda-z)-\cosh b(\Lambda-z)]\} ;
\end{aligned}
$$

then, using the Inversion Integral [6] the inverse transform of $w(z, s)$ formally will be

$$
\tilde{W}(z, \tau)=\tilde{W}_{0}(z)+\sum_{n=1}^{\infty} \frac{M\left(z, s_{n}\right)}{s_{n} \dot{D}\left(s_{n}\right)} \exp \left(s_{\mathrm{n}} \tau\right),
$$




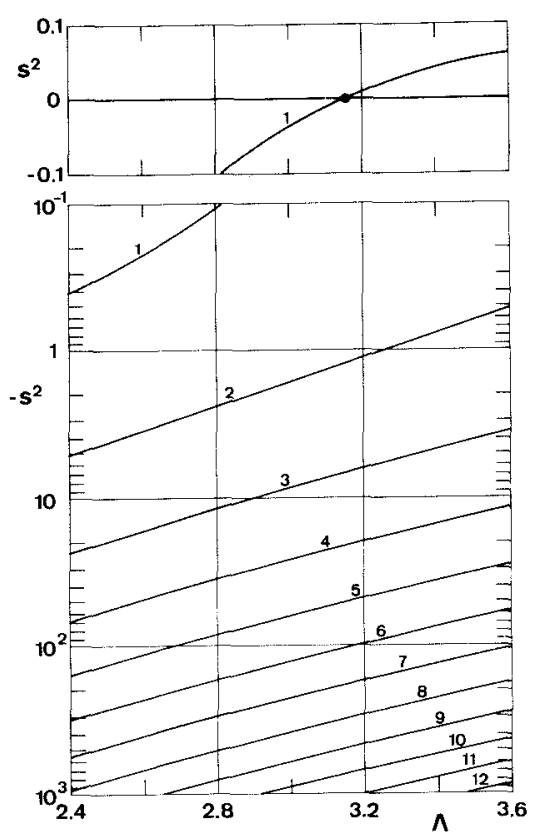

Fig. 2. Variation with the slenderness, $A$, of the roots of $D(s)=0$. Numbers on the curves indicate the root index.

where $s_{n}$ stands for the poles of $w(z, s)$ and

$\dot{\mathrm{D}}\left(\mathrm{S}_{\mathrm{n}}\right)=\left.\frac{\mathrm{d} D(s)}{\mathrm{d} s}\right|_{s=s_{n}} ;$ where

$\frac{\mathrm{d} D(s)}{\mathrm{d} s}=\frac{8 s}{a b}\left[\Lambda b\left(1+\frac{2}{b^{2}-a^{2}}\right) \cosh 2 \mathrm{a} \Lambda \sinh 2 b \Lambda\right.$

$$
+A a\left(1+\frac{2}{a^{2}-b^{2}}\right) \sinh 2 a A \cosh 2 \mathrm{~b} A
$$$$
+\cosh 2 a A \cosh 2 b A-1] \text {; }
$$

and, since the function $w(z, s)$ has a simple pole at $s=0$,

$\tilde{W}_{0}(z)=\lim _{s \rightarrow 0} s w(z, s)=\frac{1}{2}\left(1+\frac{z \cos \Lambda-\sin z}{\Lambda \cos \Lambda-\sin \Lambda}\right)$.

The poles $s_{n}$ are the roots of equation $D(s)=0$, which is the same than that already solved in[8]. The variation with the slenderness $A$ of these roots close to $A=\pi$ is shown in Fig. 2; as it can be observed there are two possibilities depending upon the value of $A$; if $A<\pi$ all roots are imaginary $s_{n}= \pm i \omega_{n}$ whereas $\Lambda>\pi$ means two real roots $s_{1}= \pm \gamma(\gamma \leqslant 1 / 2)$ the remaining roots being imaginary $s_{n}= \pm i \omega_{n}, n>2$ (observe that $s=0$ is a solution of $D(s)=0$ no matter the value of $A$ is). Then, taking into account that the roots (19) of the characteristic eqn (20) are one real, $a^{2}>0$, and the other one imaginary, $b^{2}<0$, when $s_{n}$ is imaginary, and that these roots are both imaginary when $s$ is real, plus the symmetry properties of functions $M$ and $D[M(z, s)=M(z,-s), \quad D(s)=-D(s)]$, eqn $(25)$ becomes

$$
\begin{aligned}
\tilde{W}(z, \tau)= & \frac{1}{2}\left(1+\frac{z \cos \Lambda-\sin z}{\Lambda \cos \Lambda-\sin \Lambda}\right) \\
& +2 S_{\pi}(\Lambda) \frac{M(z, \gamma)}{\gamma \dot{D}(\gamma)} \cosh \gamma \tau \\
& +2\left(1-S_{\pi}(\Lambda)\right) \frac{M\left(z, i \omega_{1}\right)}{i \omega_{1} \dot{D}\left(i \omega_{1}\right)} \cos \omega_{1} \tau \\
& +2 \sum_{n=2}^{\infty} \frac{M\left(z, i \omega_{n}\right)}{i \omega_{n} \dot{D}\left(i \omega_{n}\right)} \cos \omega_{n} \tau,
\end{aligned}
$$

where $S_{\pi}(A)$ is the step function and the functions $M(z, \gamma), D(\gamma), M\left(z, i \omega_{n}\right)$ and $D\left(i \omega_{n}\right)$ are obtained from (24) and (26). Note that, in spite of its complex appearance, this expression for $\tilde{W}(z, \tau)$ is real.

On the other hand, liquid bridge interface shape is calculated from continuity eqn (7) yielding

$$
\begin{aligned}
\tilde{R}(z, \tau)= & -\frac{1}{4} \frac{\cos \Lambda-\cos z}{\Lambda \cos \Lambda-\sin \Lambda} \\
& -S_{\pi}(\Lambda) \frac{M_{z}(z, \gamma)}{\gamma^{2} \dot{D}(\gamma)} \sinh \gamma \tau \\
& -\left(1-S_{\pi}(\Lambda)\right) \frac{M_{z}\left(z, i \omega_{1}\right)}{i \omega_{1}^{2} \dot{D}\left(i \omega_{1}\right)} \sin \omega_{1} \tau \\
& -\sum_{n=2}^{\infty} \frac{M_{z}\left(z, i \omega_{n}\right)}{i \omega_{n}^{2} \dot{D}\left(i \omega_{n}\right)} \sin \omega_{n} \tau .
\end{aligned}
$$

Once the response of the system to the step function has been calculated, the response to some prescribed injection law can be obtained using the Duhamel's Formula.

\section{CONCLUSION}

In spite of the relative simplicity of the mathematical model here used the results obtained show the main characteristics of the filling-withdrawal process. For instance, when the slenderness of the initially cylindrical liquid column is greater than the stability limit $(\Lambda>\pi)$ a time exponential term appears, see eqn (28), which indicates the unstable behavior of such fluid configurations. Certainly, as the study here presented is based on a linear approximation, it does not yield any information on how the stability limit becomes modified by the pressure field induced by liquid injection. However, this study is a first step in the analysis of the filling-withdrawal problem.

\section{REFERENCES}

1. D. Langbein, Liquid and interfaces. In Results of Spacelab-1, ESA SP-222, pp. $443-447$ (1984).

2. I. Martinez and A. Sanz, Long liquid bridges aboard sounding rockets. ESA J. 9, 323-328 (1985).

3. J. Meseguer and A. Sanz, Numerical and experimental study of the dynamics of axisymmetric slender liquid bridges. J. Fluid Mech. 153, 83-101 (1985).

4. A. Sanz, The influence of the outer bath in the dynamics of axisymmetric liquid bridges, J. Fluid Mech. 156, $101-140(1985)$ 
5. J. Meseguer, A. Sanz and J. López, Liquid bridge breakages aboard Spacelab-D1. J. Crystal Growth $\mathbf{7 8}$ 325-334 (1986).

6. R. V. Churchill, Operational Mathematics, 2nd edn McGraw-Hill, New York (1958).
7. H. S. Carslaw and J. C. Jaegar, Operational Methods in Applied Mathematics, 2nd edn. Dover, New York (1963).

8. J. Meseguer, The breaking of axisymmetric slender liquid bridges. J. Fluid Mech. 130, 123-151 (1983). 